\title{
One Case about the Diagnosis and Treatment of Right-Sided Infective Endocarditis without Any Inducement
}

\author{
Shengsen Chen*, Kangkang Yu*, Qingxia Ling, Chong Huang, Jianming Zheng, Qi Cheng, \\ Mengqi Zhu, Ning Li ${ }^{\#}$, Mingquan Chen\# \\ Department of Infectious Diseases, Huashan Hospital, Fudan University, Shanghai, China \\ Email: "liningapple@126.com, " $\underline{\text { mingquanchen@fudan.edu.cn }}$
}

Received 30 October 2014; revised 25 November 2014; accepted 5 December 2014

Copyright (C) 2014 by authors and Scientific Research Publishing Inc.

This work is licensed under the Creative Commons Attribution International License (CC BY).

http://creativecommons.org/licenses/by/4.0/

(c) (i) Open Access

\begin{abstract}
We present a case of Staphylococcus aurous tricuspid valve endocarditis without any risk factors (intravenous drug users, with pacemaker or central venous lines and with congenital heart disease). Transthoracic echocardiography, as first line examination, showed the vegetations on tricuspid valve. In our case, the diagnosis and treatment of right-sided infective endocarditis without any inducement were extremely difficult. Therefore, once a patient comes out with fever, the blood culture remains positive for gram-positive bacterium, and the infectious location still unknown, then the infective endocarditis should be considered. In addition, surgical measure is also an important therapy for right-sided infective endocarditis.
\end{abstract}

\section{Keywords}

Right-Sided Endocarditis, Staphylococcus aureus, Daptomycin, Vancomycin

\section{Introduction}

Portal of entry, implanted foreign material, and repaired congenital heart defects were the main risk factors of right-sided infective endocarditis and vegetation size on the right-sided valves was much smaller than those beyond the valves [1]. Nevertheless, there still exists some right heart endocarditis without any predisposing factors. Here, we showed one case about tricuspid valve endocarditis without any obvious risk factors and this case treated with daptomycin.

\footnotetext{
*These authors contributed equally to this work.

\#Corresponding authors.
}

How to cite this paper: Chen, S.S., Yu, K.K., Ling, Q.X., Huang, C., Zheng, J.M., Cheng, Q., Zhu, M.Q., Li, N. and Chen, M.Q. (2014) One Case about the Diagnosis and Treatment of Right-Sided Infective Endocarditis without Any Inducement. Case Reports in Clinical Medicine, 3, 631-635. http://dx.doi.org/10.4236/crcm.2014.312134 


\section{Case Report}

A 14-year-old boy came out fever on December 14, 2013, the highest body heat was $37.9^{\circ} \mathrm{C}$, together with chilly and shiver. The patient also reported some headaches and malaise but denied any chest pain, shortness of breath, cough, expectoration, nausea, emesis, abdominal pain, diarrhea, or other symptoms. Then he went to the local hospital, and took some laboratory test immediately. For a while, the laboratory test results were as follows: white blood cell count $9.3 \times 10^{9} / \mathrm{L}$, with $80.1 \%$ neutrophils; blood culture showed positive for Staphylococcus aureus, thus the erythromycin and piperacillin-tazobactam were administered to resist the bacteria. About two weeks later, patient's temperature became normal and blood culture showed negative result. On December 31, 2013 , the boy came out fever again, with a body temperature of $39^{\circ} \mathrm{C}$, immediately, he was taken to pneumology department of Huashan Hospital. The blood reexamination then were as follows: blood cell count $8.51 \times$ $10 \%$ L, neutrophils account for 82.1\%; blood culture presented positive for Staphylococcus aureus; whereas, echocardiogram and autoimmune indicators were normal, so the teicoplanin was used; however, body temperature still hard to control. Ultimately, the boy was transfered to the department of infectious disease in Huashan Hospital.

On January 14, 2014, blood tests showed a haemoglobin level of $112 \mathrm{~g} / \mathrm{L}$, white cell count of $6.21 \times 10^{9} / \mathrm{L}$, platelet count of $222 \times 10^{9} / \mathrm{L}$, and a C reactive protein (CRP) level of $25.4 \mathrm{mg} / \mathrm{L}$. So all antibiotics were stopped, and we observed patient's fever type for three days. On January 17, 2014, we heard murmurs of his heart and the blood culture remained positive for gram-positive bacterium, and fever persisted. Therefore, combination therapy with vancomycin $1000 \mathrm{mg}$ intravenously q12 h and doxycycline $100 \mathrm{mg}$ intravenously bid was administered. On January 18, 2014, the organism was identified as Staphylococcus aureus by blood culture (Figure 1), then the doxycycline was withdrawed. However, six days later, the boy's temperature failed to be controlled, and the results of transthoracic echocardiography were as follows: 1) a mobile mass of $6 \mathrm{~mm} \times 6$ mm was attached to the tricuspid valve (Figure 2(a)); 2) a moderate tricuspid regurgitation with flow toward the side wall of right atrium (Figure 2(b)); 3) the septal cusp of the tricuspid valve prolapsed. Finally, the diagnosis of endocarditis on tricuspid valve was made. According to the drug sensitive test, vancomycin was used for ten days, but the patient still remained high temperature (about $39.2^{\circ} \mathrm{C}$ ). Given that daptomycin is a novel cyclic lipopeptide with activity against most aerobic Gram-positive pathogens, as a result, daptomycin 500 mg intravenously once daily was started and vancomycin was stopped. Over the following days, body temperature was well controlled,

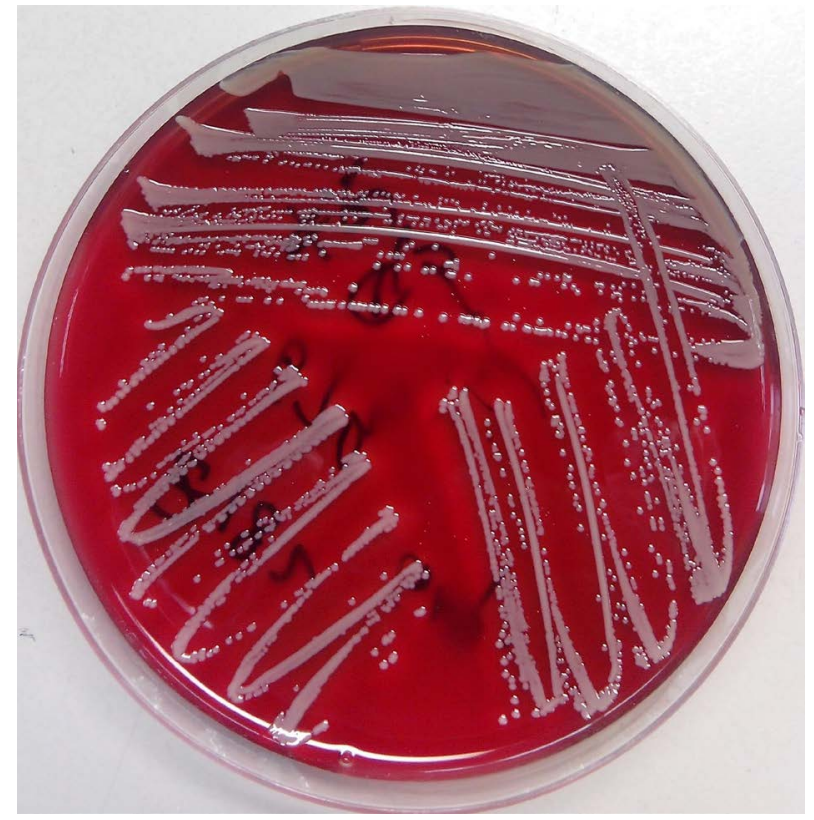

Figure 1. Growth of Staphylococcus aureus after 24 hours on blood agar and aerobic incubation at $37^{\circ} \mathrm{C}$ showing shiny, convex, haemolytic white colonies $2-3 \mathrm{~mm}$ in diameter after $48 \mathrm{~h}$. 

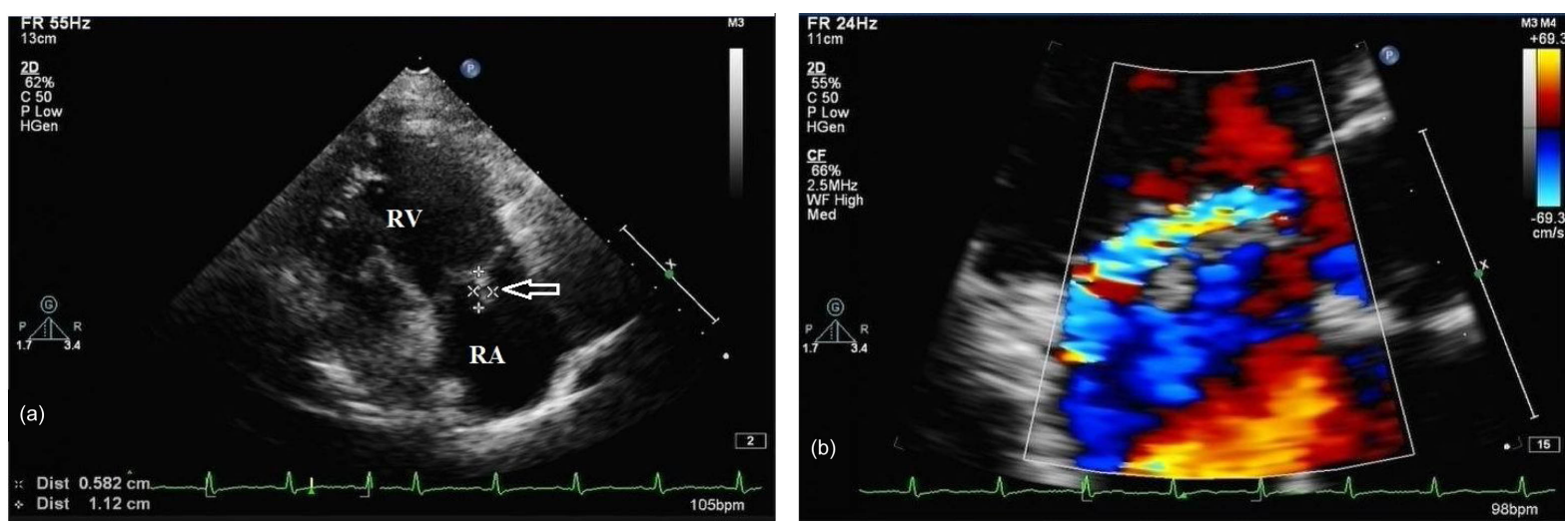

Figure 2. (a) Transthoracic echocardiographic view showing a vegetation $(6 \mathrm{~mm} \times 6 \mathrm{~mm})$ on tricuspid valve (indicated by arrow); (b) Color flow doppler echocardiogram showing a moderate tricuspid regurgitation with flow toward the side wall of right atrium.

ranging from $36^{\circ} \mathrm{C}$ to $37.8^{\circ} \mathrm{C}$. Unfortunately, the reexamination of echocardiography showed that the vegetation became larger than before (Figure 3) on February 20, 2014. We got that the antibiotics failed to clear the vegetation; therefore, the surgical measure must be taken.

On February 28, 2014, the patient transferred to Shanghai Chest Hospital for further therapy. Blood culture showed a negative result, and on 3 March 2014 the echocardiography was examined again, which revealed that a mobile mass of $15 \mathrm{~mm} \times 9 \mathrm{~mm}$ attached to the tricuspid valve became larger than the vegetation reported on February 20, $2014(11 \mathrm{~mm} \times 5 \mathrm{~mm})$. Three days later, the tricuspid valve annuloplasty was taken under general anesthesia with extracorporeal circulation, the size of vegetation was $15 \mathrm{~mm} \times 10 \mathrm{~mm}$ seen in the operation. On March 17, 2014, the echocardiography after surgical operation showed that the mobile mass attached to the tricuspid valve disappeared. After two weeks, the boy recovered well and was released from hospital.

We followed up the patient for six months, his body temperature and blood routine test kept normal, and no vegetation was found on tricuspid valve again.

\section{Discussion}

In this case, the patient got right-sided infectious endocarditis without any inducement (like portal of entry, implanted foreign material, congenital heart defects and intravenous drug use), and the first echocardiogram was normal (during the time treated in the pneumology department of Huashan Hospital). According to the fever, the result of blood culture (positive for Staphylococcus aureus) of blood culture and the rise of white blood cell count, we can conclude that the bacterial infection existed, but the infective location remained unknown. On January 24, 2014 the reexamination of echocardiogram showed a mobile mass attached to the tricuspid valve, then the right-sided infective endocarditic was diagnosed. For this patient, the diagnosis of right-sided infectious endocarditic is extremely hard.

As is well known to us, vancomycin is a standard medication for infectious endocarditic infected by Staphylococcus aureus [2]. However, vancomycin was stopped in our case for the possibility of drug fever. The reasons we considered drug fever as follows: 1) except the heart, no other infectious locations identified; 2) drug sensitive test showed that Staphylococcus aureus from the patient sensitive to vancomycin; 3) although the body temperature beyond normal, patient's condition seemed well and the toxic symptoms rarely seen from him; 4) from January 17 (vancomycin started) to January 26 (vancomycin stopped), the body temperature often exceeded the normal level, but the blood routine examination showed few information about bacterial infection (Table 1).

Daptomycin is a novel cyclic lipopeptide with activity against most aerobic Gram-positive pathogens, including strains resistant to vancomycin [3] [4]. In a randomized clinical trial, daptomycin was as effective as a control regimen (low-dose gentamicin plus vancomycin) in patients with bacteremia caused by S. aureus, including the subset with right-sided endocarditis [2] and those with methicillin-resistant strains [5]. After initiation of daptomycin to the treatment regimen in this patient, resolution of fever and pervasive bacteremia was attained. Nevertheless, the vegetation attached to the tricuspid valve still became larger, finally, the surgical measure must 


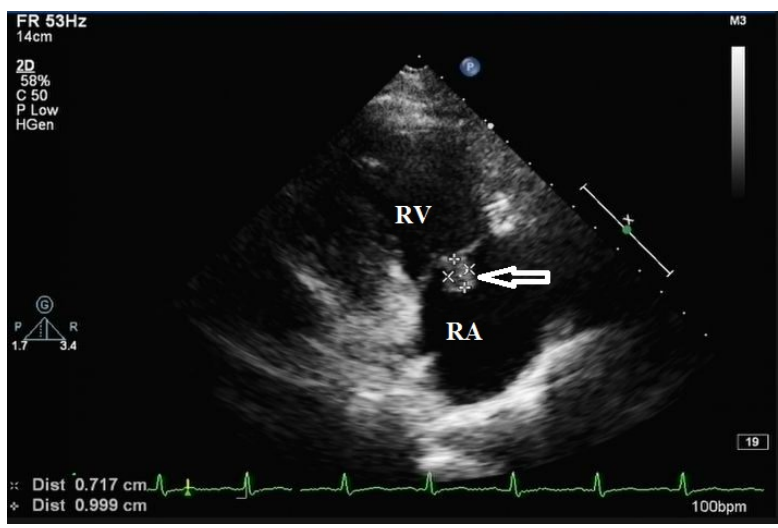

Figure 3. Transthoracic echocardiographic view showing mobile vegetation $(11 \times 5 \mathrm{~mm})$ attached to tricuspid valve (indicated by arrow), which became larger than former one $(6 \mathrm{~mm} \times 6$ $\mathrm{mm})$.

Table 1. The results of blood routine test.

\begin{tabular}{cccccc}
\hline Date & WBC & NEUT\% & LY\% & MONO $\%$ & NEUT \\
\hline $2014-01-16$ & $6.13 \times 10^{9} / \mathrm{L}$ & 77.0 & 15.8 & 6.2 & $4.72 \times 10^{9} / \mathrm{L}$ \\
$2014-01-17$ & $7.18 \times 10^{9} / \mathrm{L}$ & 76.6 & 14.8 & 7.9 & $5.50 \times 10^{9} / \mathrm{L}$ \\
$2014-01-26$ & $7.48 \times 10^{9} / \mathrm{L}$ & 68.1 & 18.0 & 8.7 & $5.09 \times 10^{9} / \mathrm{L}$ \\
\hline
\end{tabular}

be taken. The reasons leading to therapeutical failure of daptomycin mainly are as follows: 1) the vegetation attached to the valvula tricuspidalis is a foreign matter without blood supply, so the antibiotic hardly permeate into it and rarely to kill bacteria; 2) because the extremely large size of vegetation, daptomycin hardly exert its effect to kill bacteria; 3) the patient was treated with daptomycin at a dose of $500 \mathrm{mg}$ (6 mg/kg daily [2]) daily; however, the patient's weight is $95 \mathrm{~kg}$, strictly speaking, the daily dose of daptomycin must be $570 \mathrm{mg}$, so 500 mg daptomycin daily may not be enough to kill bacteria in the vegetation; 4) the abscess might be formed in tricuspid valve, so the blood concentration of daptomycin in this location was insufficient to eliminate the vegetation.

The diagnosis of infectious endocarditis was mainly depend on the Duke standards [6]. In this case, the patient got right-sided infectious endocarditis without any risk factors (like portal of entry, implanted foreign material, congenital heart defects and intravenous drug use), and the first echocardiogram is normal. The information about bacterial infection were merely fever, blood culture just showed positive for Staphylococcus aureus, but the locations of infection remained unknown. Thereby, the clinical manifestation of this case rarely matched with the Duke standards, and it's really difficult to diagnose the right-sided infectious endocarditis for this patient. The body temperature was controlled after the daptomycin initiated, but the vegetation attached to the tricuspid valve still became larger, at last, the patient had to take surgical treatment.

\section{Conclusion}

According to this case, we can get some enlightenments as follows: 1) once a patient comes out with fever, the blood culture remains positive for gram-positive bacterium, and the infectious location still unknown, the infective endocarditis should be considered; 2) when the infective endocarditis is identified, the blood culture show that Staphylococcus aureus is positive, if the bacterium is sensitive to vancomycin, vancomycin should be firstly considered. The daptomycin may be considered in the condition that Staphylococcus aureus is resistant to vancomycin or the patient is allergic to the vancomycin; 3) when the growth of vegetation is hardly controlled, the patient must firmly turn to surgeons for help, because medical treatment alone is hard to clear the stubborn vegetation. Finally, sharing with this case, we hope more clinicians can get some help from it about the diagnosis and treatment of right-sided infectious endocarditis without any induced factors.

\section{Acknowledgements}

The authors of this manuscript are grateful to Dr. Zhu hui who provided the echocardiogram and Dr. Liu qin 
who provided picture of blood culture, Huashan Hospital, Shanghai, China.

\section{References}

[1] Yuan, S.M. (2014) Right-Sided Infective Endocarditis: Recent Epidemiologic Changes. International Journal of Clinical and Experimental Medicine, 7, 199-218.

[2] Fowler, V.J., Boucher, H.W., Corey, G.R., Abrutyn, E., Karchmer, A.W., Rupp, M.E., et al. (2006) Daptomycin versus Standard Therapy for Bacteremia and Endocarditis Caused by Staphylococcus aureus. New England Journal of Medicine, 355, 653-665. http://dx.doi.org/10.1056/NEJMoa053783

[3] Steenbergen, J.N., Alder, J., Thorne, G.M. and Tally, F.P. (2005) Daptomycin: A Lipopeptide Antibiotic for the Treatment of Serious Gram-Positive Infections. Journal of Antimicrobial Chemotherapy, 55, 283-288. http://dx.doi.org/10.1093/jac/dkh546

[4] Sader, H.S., Streit, J.M., Fritsche, T.R. and Jones, R.N. (2004) Antimicrobial Activity of Daptomycin against Multidrug-Resistant Gram-Positive Strains Collected Worldwide. Diagnostic Microbiology and Infectious Disease, 50, 201204. http://dx.doi.org/10.1016/j.diagmicrobio.2004.07.002

[5] Rehm, S.J., Boucher, H., Levine, D., Campion, M., Eisenstein, B.I., Vigliani, G.A., et al. (2008) Daptomycin versus Vancomycin plus Gentamicin for Treatment of Bacteraemia and Endocarditis Due to Staphylococcus aureus: Subset Analysis of Patients Infected with Methicillin-Resistant Isolates. Journal of Antimicrobial Chemotherapy, 62, 14131421. http://dx.doi.org/10.1093/jac/dkn372

[6] Pierce, D., Calkins, B.C. and Thornton, K. (2012) Infectious Endocarditis: Diagnosis and Treatment. American Family Physician, 85, 981-986. 
Scientific Research Publishing (SCIRP) is one of the largest Open Access journal publishers. It is currently publishing more than 200 open access, online, peer-reviewed journals covering a wide range of academic disciplines. SCIRP serves the worldwide academic communities and contributes to the progress and application of science with its publication.

Other selected journals from SCIRP are listed as below. Submit your manuscript to us via either submit@scirp.org or Online Submission Portal.
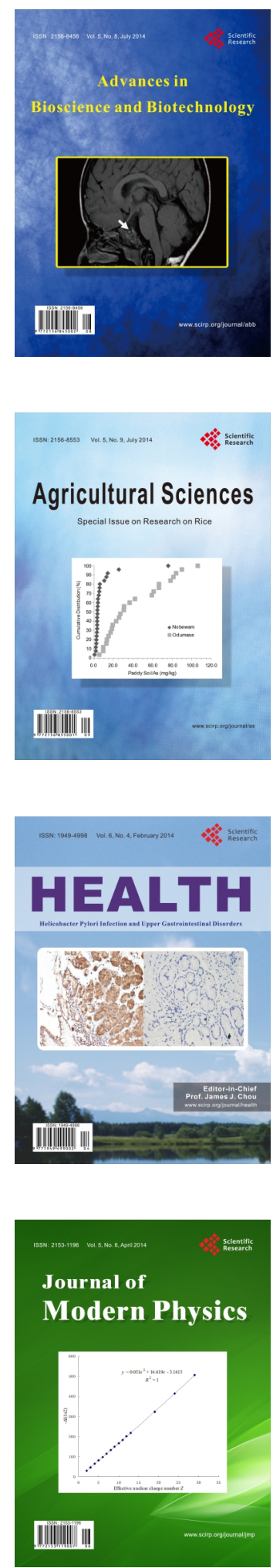
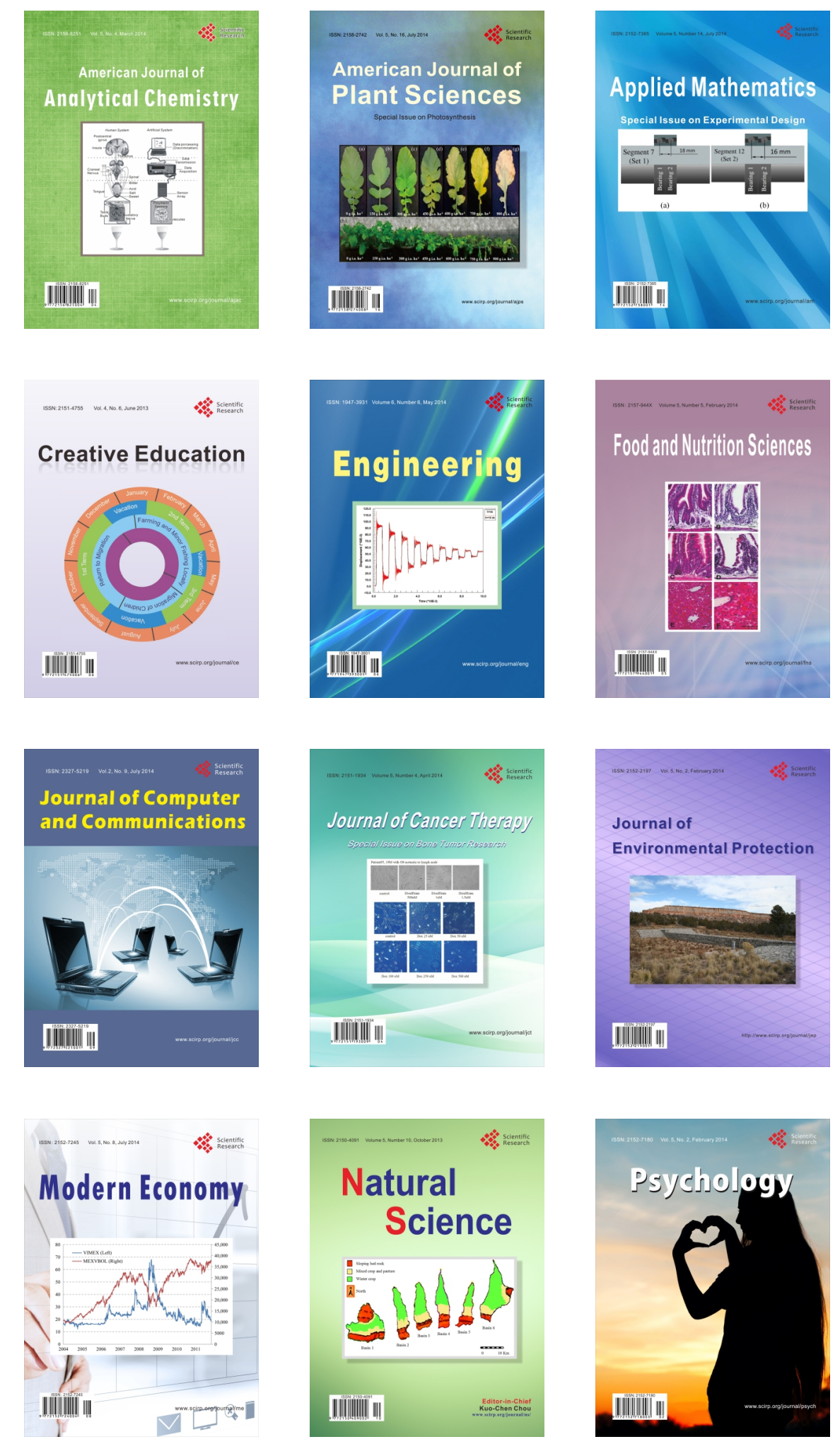